\title{
O possível efeito de mudanças climáticas e suas incertezas sobre afluências em sistemas de recursos hídricos
}

\section{The possible effect of climate changes and their uncertainties on inflows in water resources systems}

\section{Andre Schardong, Slobodan P. Simonovic, Joaquin I. B. Garcia}

Submissão: 24/11/2014

Revisão: $15 / 12 / 2014$

Aprovado: 28/12/2014
RESUMO: Nas recentes décadas, tem sido observado um constante e gradual aumento da temperatura média do planeta. Apesar de controverso, o fenômeno é atribuído diretamente a ação antrópica e à emissão de gases de efeito estufa. $\mathrm{O}$ aumento da temperatura média é considerado o gatilho para os processos de alteraçóes extremas no regime de precipitaçóes e outras variáveis atmosféricas. A precipitação, por sua vez, é componente do ciclo hidrológico, que juntamente com algumas outras variáveis como vegetação e solo, são determinantes da disponibilidade hídrica de uma bacia hidrográfica através do escoamento superficial e a recarga do solo. Neste artigo é apresentada uma metodologia que procura de forma simplificada, analisar os resultados dos modelos de circulação global (GCM) preparados para o quinto relatório de situação (AR5) do "Intergovernmental Panel on Climate Change" - IPCC. A metodologia consiste em corrigir as séries de precipitação dos modelos utilizando técnicas de "downscaling" e correção de viés. Mais especificamente, séries de precipitaçóes mensais históricas observadas são corrigidas utilizando os períodos de controle e as projeçôes para os vários cenários futuros dos modelos GCM através de ajuste e correlação de distribuição empírica de probabilidades. A correlação estatística entre o período de controle ou base dos GCM e os cenários futuros, é realizada de tal forma que a não estacionaridade das séries de precipitação, naturalmente induzidas pelas mudanças no clima, sejam adequadamente consideradas. As séries de precipitação estatisticamente corrigidas e obtidas para os cenários futuros são utilizadas para a geração das séries de vazão média natural através de um modelo chuva-vazao, calibrado com dados observados. Como estudo de caso, são utilizadas as principais bacias dos reservatórios constituintes do Sistema Cantareira, um importante contribuinte para o abastecimento da Região Metropolitana de São Paulo - RMSP, responsável pelo fornecimento de aproximadamente $33 \mathrm{~m} 3 / \mathrm{s}$. $\mathrm{Na}$ análise, foram considerados modelos com dados mensais disponíveis para três cenários futuros, discutidos e apresentados no artigo. Os resultados mostram uma grande incerteza no afluxo de vazóes com tendência de aumento na afluência média para a bacia dos reservatórios Jaguari/Jacareí e diminuição nos outros reservatórios do sistema. É brevemente discutida também o aumento do risco de falhas na garantia com comparaçáo dos resultados com a atual crise hídrica pela qual passa o Sistema Cantareira.

PALAVRAS-CHAVE: disponibilidade hídrica, modelos de circulação global, modelagem matemática
ABSTRACT: In recent decades there has been a constant, gradual rise in the average temperature of the planet. Although somewhat controversial, these phenomena are directly linked to human activity and the emission of gases responsible for the so called greenhouse effect. The rise of the average temperature is believed to be responsible for the changes in the extreme precipitation patterns and other atmospheric variables. Precipitation is an important component of the hydrological cycle that together with other variables such as land use and vegetation affects water availability in a watershed by influencing runoff and underground recharge. This paper presents a simplified methodology that aims at the analysis of several global circulation models (GCM) prepared for the $5^{\text {th }}$ Assessment Report of the "Intergovernmental Panel on Climate Change" - IPCC. The methodology consists in downscaling and bias correcting the precipitation series for the future scenarios of the GCMs based on the control or historical runs and observed data using empirical probability distributions. Bias correction is performed in a way that will adequately represent the non-stationarity of the precipitation series naturally introduced by climate changes. The bias corrected series for the future are used for generating the inflow series using a rainfall runoff model. The main watersheds of the Cantareira System Reservoirs were used as a case study. The system is responsible for providing more than $50 \%$ of the water for the Sao Paulo Metropolitan Region (RMSP), approximately $33 \mathrm{~m}^{3} / \mathrm{s}$. In the analysis monthly data from the GCM models were used for three future scenarios. Results show high uncertainty for the inflow series with a trend toward increasing average flow for the Jaguari/Jacarei reservoir and a decrease for the others. A brief discussion comparing results from the climate models with the current drought is also presented.

KEYWORDS: water availability, global circulation models, mathematical modeling. 


\section{INTRODUÇÃO}

Recentes estudos do IPCC (Painel Intergovernamental sobre Mudanças Climáticas) indicam um provável aumento da temperatura e precipitaçáo no planeta no século 21 . As projeçôes nas variaçôes não são uniformes, e enquanto algumas regiōes experimentarão um aumento significativo nos níveis de precipitaçôes, outras deverão sofrer com o declínio em relação aos níveis históricos.

Segundo o último relatório de avaliação do IPCC Assessment Report 5 (AR5, IPCC, 2014), o aumento gradual da temperatura, que presumidamente ocorrerá na maioria das regiôes do planeta, é atribuído especialmente aos níveis de gases de efeito estufa (dióxido de carbono, metano e óxido de nitrogênio). Em valores percentuais, há indicação de variação positiva na temperatura média do planeta de 0,3 à $4,8 \%$ até o final de 2100 em relação ao período histórico observado(TAYLOR et al., 2012; KHARIN et al., 2013; IPCC, 2014).

$\mathrm{O}$ incremento na temperatura média do planeta tem sido diretamente relacionado à variação do regime de precipitação com comportamento distinto em diferentes regiōes (WILCOX et al., 2007; ALLAN et al., 2008, SOLAIMAN et al., 2011). Em regióes de latitudes mais elevadas, existe expectativa de aumento significativo dos níveis de precipitação em relação aos valores históricos. As regióes próximas aos trópicos, áridas e semiáridas, muito possivelmente notarão incremento menor ou até reduçáo dos níveis de precipitação (IPCC, 2007 e 2014).

A América do Sul deverá sofrer também com variaçóes significativas. No Nordeste Brasileiro, é estimada variação negativa de até $30 \%$ nos níveis de precipitação atual. Já na região Sudeste e na Bacia do Prata, a variação esperada nos níveis de precipitação é menos acentuada, podendo variar negativamente em algumas regióes e até positivamente em até $10 \%$ em outras (MARENGO et al., 2011).

Os cenários futuros são gerados em função de estimativas a partir dos resultados de modelos de circulação global (GCMs) ou modelos de circulação acoplados atmosfera-oceano (AOGCMs). Diferentes modelos e resultados estão disponíveis na literatura e são produzidos por diferentes centros de pesquisa. Estes modelos de circulação global simulados para o IPCC AR5 são baseados em cenários definidos por diferentes perspectivas de desenvolvimento econômico e social futuro que afetam ou determinam os níveis de emissóes de gases de efeito estufa. Os modelos do Coupled Model Inter-Comparison Phase 3 (CMIP3) preparados para o IPCC AR4, baseados no SRES (Special Reporton Emissions Scenarios) foram produzidos considerando três cenários principais: $\mathrm{A} 2$ de alta emissão, B2de baixa emissão e o A1B de média emissão (NAKICENOVIC et al. 2000).Para os modelos do CMIP5 preparados para o AR5, os cenários A2, B2 e A1B foram substituídos pelos chamados RCPs (Representative Concentration Pathways) e foram projetados de forma a acomodar uma gama de possibilidades de desenvolvimento econômico e social (TAYLOR et al. 2012; KHARIN et al., 2013). Três RCPs principais são utilizados para as simulaçóes futuras RCP 2.6, RCP 4.5 e RCP 8.5. O número associado ao RCP é o valor do fluxo de calor ou radiativo ao final do século XI em Watts $/ \mathrm{m}^{2}$, o que equivale respectivamente, a um nível de emissão de CO2 de 490 p.p.m (partes

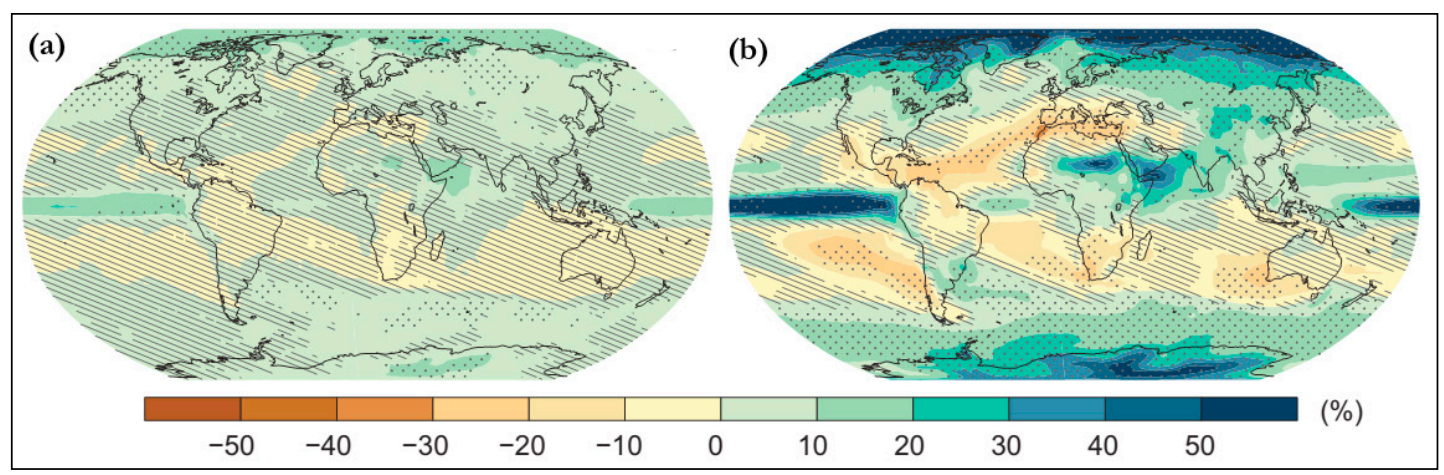

FIGURA 1. Variação estimada da precipitação no planeta em relação aos níveis históricos em \%. Fonte: IPCC (2014). 
por milhão), 650 p.p.m e 1370 p.pm. (VUUREN et al., 2011 e WCRP, 2011). O cenário RCP 4.5 é o cenário desejado, ou seja, aquele em que os países consigam controlar os níveis de emissóes, e o nível de CO2 na atmosfera se estabilize logo após 2100. Entretanto, o cenário RCP 8.5 é reconhecidamente o mais provável. As rodadas de controle (simulação do período histórico) são realizadas, em geral para a maioria dos modelos de 1850 a 2005.

Atualmente, os modelos climáticos de circulação global ou modelos regionais são umas das melhores ferramentas para determinar o impacto na disponibilidade hídrica futura em bacias hidrográficas e/ ou sistemas de recursos hídricos em geral. Gaur e Simonovic (2013) utilizaram GCMs para determinar o impacto de mudanças no clima para uma bacia hidrográfica na bacia do Rio Grant, Ontario - Canada. A metodologia consiste em conversão das séries de precipitação em escala local e posterior utilização em modelo chuva-vazão distribuído. Solaiman e Simonovic (2011) e Das e Simonovic (2012), Eum e Simonovic (2012) analisaram o possível impacto em séries de vazóes utilizando os GCMs preparados para o IPCC AR4 para a cidade de London, Canada. Um algoritmo estocástico K-NN ("k"iésimo vizinho mais próximo), Sharif e Burn (2007) e King et al. 2013, para a geração das séries sintéticas de vazão dos cenários futuros. Bowering et al. (2013) utilizam metodologia similar para avaliação do impacto de mudanças climáticas em forma de mapa de risco de inundaçóes. Bravo et al. (2013) utilizaram também modelos de circulação global do IPCC AR4 para analisar o impacto das possíveis mudanças climáticas no regime de vazóes na bacia do rio Alto Paraguai.

Para a utilização dos resultados disponibilizados pelos GCMs em escala local, é necessário realizar a transformação. A resolução espacial destes modelos varia, sendo geralmente compostos por pontos de grade representando os elementos do modelo matemático utilizado e, aos quais séries temporais estáo associadas. A resolução das séries temporais também é variada podendo ser mensal, diária ou até mais detalhada. Várias técnicas existem e são discutidas em Salathé $\mathrm{Jr}$ et al (2007), Piani et al. (2010) e Gaur e Simonovic (2013).

O objetivo deste artigo é a análise do possível impacto na disponibilidade hídrica de um sistema produtor de água para abastecimento público, utilizando como base as séries de precipitaçáo disponibilizadas pelos modelos climáticos de circulação global (GCM). Como estudo de caso é utilizado um dos sistemas que abastece a região metropolitana de
Sáo Paulo (RMSP). RMSP é abastecida por 6 grandes sistemas produtores e atualmente passa por sua pior crise hídrica devido aos baixos níveis de precipitação registrados no veráo de 2013 e todo ano de 2014 . O sistema Cantareira é o mais importante e responsável pelo abastecimento de cerca de 50\% da RMSP e está com os níveis mais baixos já registrados. Medidas drásticas como utilizaçáo de volume morto estão sendo adotadas para garantir o abastecimento. O objetivo principal do artigo é analisar as séries de precipitação extraídas dos modelos climáticos e compará-los às séries históricas observadas de postos pluviométricos. Um modelo chuva-vazão é aplicado para possibilitar também a análise das séries de vazóes afluentes aos principais reservatórios do Sistema.

Na próxima seção do artigo, é apresentada a metodologia aplicada, a descrição do estudo de caso e os resultados obtidos, seguido das conclusóes.

\section{METODOLOGIA}

A metodologia consiste em inicialmente extrair as séries de precipitaçáo total mensal dos postos das bacias do estudo de caso e as séries dos modelos GCM. Em todas as séries extraídas dos modelos GCM, bem como nas séries observadas, uma função empírica de probabilidades é ajustada. Com a distribuição de probabilidades ajustada, a correção de viés é realizada através da correlação dos cenários futuros, cenário de controle e as séries observadas. As séries de precipitaçôes corrigidas para os cenários futuros são aplicados em um modelo de transformação chuva-vazão para determinar as séries afluentes aos reservatórios. Por fim, um modelo de otimização é aplicado para verificar a garantia de fornecimento mediante às séries de vazóes determinada.

As etapas para a avaliação do impacto são listadas a seguir:

1) Extração dos dados do modelo GCM

2) Transformação/interpolação das séries de precipitação dos modelos GCM em escala local para cada sub-bacia do sistema;

3) Aplicação da correção de viés nas séries de precipitação para os cenários futuros;

4) Ajuste dos parâmetros do modelo Chuva vazão SMAP através de calibração semiautomática com auxílio de modelo de otimização;

5) Aplicação da série de precipitação no modelo chuva-vazão SMAP para geração de série de vazões futuras; 
Um fluxograma da metodologia descrita é apresentado na Figura 2. Os passos da metodologia são descritos detalhadamente a seguir.

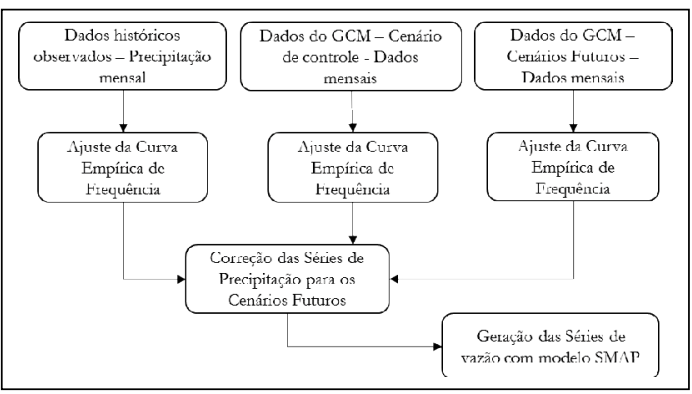

FIGURA 2. Fluxograma da Metodologia de Atualização das Séries de Vazão.

As séries de precipitação mensal extraída dos modelos GCM são convertidos em escala local (bacia hidrográfica) utilizando a interpolação de quatro pontos da grade do modelo mais próximos à estação com os dados observados. O método do inverso da distância foi utilizado, conforme equação (1).

$$
\begin{aligned}
& P_{g c m}^{k}=\sum_{i=1}^{4} w_{i} P_{g c m, i} \\
& w_{i}=\frac{d_{i}^{2}}{\sum_{i=1}^{4} d_{i}^{2}}
\end{aligned}
$$

onde $\mathrm{P}_{\mathrm{g} \mathrm{cm}}^{\mathrm{k}}$ é a precipitação total mensal do modelo no ponto de interesse, $\mathrm{P}_{\mathrm{g} \mathrm{cm}, \mathrm{i}}$ a precipitação no ponto de grade $i$ do modelo GCM, $\mathrm{d}_{\mathrm{i}}$ a distância do ponto de grade $i$ do GCM ao ponto de interesse e $\mathrm{w}_{\mathrm{i}}$ é o peso atribuído a cada ponto para cálculo da média ponderada.

Após a interpolação e conversão das séries de precipitação do GCM em escala local, são aplicadas as correções de viés nas séries futuras. Esta correção é de extrema importância, dada a incerteza envolvida na previsão dos modelos em relação aos cenários futuros. A escala temporal utilizada é mensal, o que permite utilizar os métodos propostos por $\mathrm{Li}$ et al. (2010) e Piani et al. (2010) que consiste em relacionar as curvas de frequência acumulada (CFA) das séries extraídas dos modelos climáticos com as séries de precipitação observadas em cada bacia hidrográfica. $\mathrm{O}$ cálculo da correção é matematicamente apresentada na equação (2).

$x_{a j u s t}=x_{m-p}+\operatorname{inv} F_{o-c}\left(F_{m-p}\left(x_{m-p}\right)\right)-\operatorname{inv} F_{m-c}\left(\left(x_{m-p}\right)\right)$

onde $\mathrm{x}_{\text {ajust }}$ é a precipitação mensal dos cenários futuros ajustada, $\mathrm{x}_{\mathrm{m}-\mathrm{p}}$ a precipitação mensal dos cenários futuros, $\mathrm{F}$ a curva de frequência acumuladadas séries de precipitação mensal, sendo o índice $\mathrm{m}$ referente à precipitaçáo do modelo, $\mathrm{p}$ representa o cenário futuro projetado, c o cenário de controle ou histórico, e o representa a série histórica observada. O termo invF representa o inverso da funçáo de probabilidades $\mathrm{F}$. Todas as unidades de precipitação são expressas em $\mathrm{mm} / \mathrm{mês}$.

Para o cálculo da CFA, foi utilizada a distribuição de frequência empírica, embora distribuições teóricas de probablidades possam ser utilizadas. De fato, autores como Li et al. (2010) e Piani et al. (2010) utilizam a distribuição Beta e Gama, respectivamente. A escolha pela utilizaçao da distribuição empírica elimina particalmente o erro introduzido na utilização de uma distribuição de probalidades, e apesar das interpolações necessárias, o método é computationalmente eficiente.

\section{O modelo chuva-vazão SMAP}

O modelo chuva-vazáo SMAP (soil moisture accounting procedure), originalmente proposto por Lopes et al. (1982), possui estrutura simples e eficaz especialmente para regióes com escassez de dados. $\mathrm{O}$ modelo é conceitual e concentrado, sendo que a estrutura é composta por três reservatórios, representando: i) o armazenamento superficial; ii) a camada superior do solo e iii) armazenamento subterrâneo, conforme Figura 3.

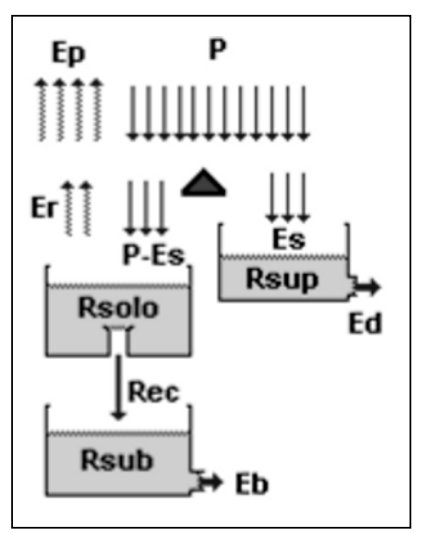

FIGURA 3.

Estrutura do modelo SMAP mensal Fonte: Lopes et al. (1982). 
O armazenamento superficial (Rsup) pode ser matematicamente escrito conforme equações (3) a (5).

$$
\begin{gathered}
\frac{d R \sup }{d t}=E s-E d \\
E s=\left\{\begin{array}{lr}
0 & \text { se } P \leq A I \\
\frac{(P-A I)^{2}}{P-A I-S} & \text { se } P>A I
\end{array}\right. \\
E d=R \operatorname{Rup}(1-K 2), \quad \text { onde } K 2=0,5^{1 / K 2 t}
\end{gathered}
$$

onde Es representa o escoamento superficial direto, obtido pelo método do Soil Conservation Service (SCS), Ed a vazão de depleção do escoamento superficial direto, $P$ a precipitação, $A I$ : abstração inicial para o método do SCS, $S$ a abstração potencial, também para o método do SCS. Ed é definido como função do nível do reservatório superficial (Rsup) e $K 2 t$ o coeficiente de depleção do reservatório superficial, como definido em Lopes (1982). Todas as unidades são expressas em $m m$, exceto por $d t$ e $K 2 t$, que possuem dimensão de tempo (mês).

O reservatório da camada superior do solo $(R s)$ pode ser matematicamente escrito conforme equaçóes (6) a (11).

$$
\begin{aligned}
& \frac{d R s}{d t}=P-E s-E r-R e c \\
& E s= \begin{cases}E p & \text { se } I N>E p \\
I N+(E p-I N) \cdot T i & \text { se } I N \leq E p\end{cases} \\
& R e c= \begin{cases}0 & \text { se } R s \leq R s M \\
\operatorname{Crec} \times T U \times(R s-R s M) & \text { se } R s>R s M\end{cases} \\
& T U=\frac{\text { Rsolo }}{\text { Str }} \\
& R s M=C a p c \times R s \\
& I N=P-E S
\end{aligned}
$$

onde $P$ e $E s$ conforme definidos acima, Er é a taxa de evapotranspiração, Rec é a recarga do reservatório do subsolo, Crec o coeficiente de recarga do reservatório do subsolo, $I N$ é definido como a diferença entre $P$ e $E s$ e é a parcela da precipitação que infiltra, $T U$ o teor de umidade do solo, STR a capacidade de campo máxima do solo e Capc a capacidade de campo máxima do solo em porcentagem (Lopes et al. 1982).
O reservatório do subsolo $(R s u b)$ pode ser escrito matematicamente conforme equaçóes (12) e (13).

$$
\begin{gathered}
\frac{d R s u b}{d t}=R e c-E b \\
E b=R s u b(1-K k t) \quad \operatorname{com} \quad K k t=0,5^{(1 / K k 0 t)}
\end{gathered}
$$

onde $E b$ a vazão do escoamento de base e $K k t$ é o coeficiente de depleção do reservatório do subsolo, como definido em Lopes et al.(1982). A vazão média final é calculada pela equação (14).

$$
Q_{M}=(E b+E d) \times C V
$$

onde CVé o coeficiente de conversão de $\mathrm{mm}$ para $\mathrm{m}^{3} / \mathrm{s}$, e $Q_{M}$ é a vazão média mensal no exutório da bacia em $\mathrm{m}^{3} / \mathrm{s}$.

Para avaliar o processo de calibração automática, uma função objetivo (FO), a soma dos erros quadráticos médios com pesos (PWRMSE), foi selecionada (CUNDERLIK; SIMONOVIC, 2005; SCHARDONG et al., 2009; USACE, 2001). Esta funçáo considera o quadrado das diferenças entre vazão observada e calculada. A cada intervalo de tempo é atribuído um peso proporcional à magnitude da vazão calculada. Assim, vazóes maiores que a vazão média observada recebem um peso maior que 1,0 , e menores que a média, um peso menor que 1,0 conforme equaçáo (15).

$$
\text { PWRMSE }=\sqrt{\frac{1}{N} \sum_{t=1}^{N}\left(Q_{o}(t)-Q_{M}(t)\right)^{2} \times \frac{Q_{o}(t)-Q_{O A}}{2 Q_{o A}}}
$$

onde: $N$ é o número de intervalos de tempo (meses neste caso), $Q_{O}(t)$ e $Q_{M}(t)$ são a vazão observada e calculada, respectivamente, $t$ intervalo de tempo, e $Q_{O A}$ é a vazão média observada.

\section{Calibração do SMAP}

Para a calibraçáo do modelo foi utilizado um algoritmo evolucionário, o Differential Evolution (DE), proposto por Price e Storn (1997). O DE é um dos algoritmos evolucionários mais recentes para solução de problemas de otimização com valores reais. $\mathrm{ODE}$ utiliza um operador de mutação simples baseado nas diferenças entre os pares de solução (também chamado de vetores) com o objetivo de encontrar a 
direção de busca com base na distribuição de soluçóes na população corrente.

Diferentemente do Algoritmo Genético, que depende fortemente do operador de cruzamento, o DE utiliza a mutaçáo como o mecanismo principal de busca, e a seleção para indicar a direção dentro da regiáo viável do espaço de decisão. Mais detalhes relacionados ao algoritmo $\mathrm{DE}$ podem ser encontrados em Price e Storn (1997), Price et al. (2005) e Schardong et al. (2011).

\section{ESTUDO DE CASO}

A disponibilidade de água per capita na regiáo metropolitana de São Paulo (RMSP) é dez vezes inferior ao mínimo estabelecido pela ONU (organização das naçôes unidas), conforme Chuva (2008). Enquanto a disponibilidade hídrica média per capita no Brasil é estima em certa de $34.000 \mathrm{~m}^{3}$ de água por pessoa por ano, na RMSP esse valor é de aproximadamente $200 \mathrm{~m}^{3}$, muito inferior mínimo de $2500 \mathrm{~m}^{3}$ por ano, considerado ideal. A baixa disponibilidade é causada especialmente pela densidade populacional elevada da região e é agravada pelos altos níveis de poluição dos rios Tietê e Pinheiros.

Atualmente seis grandes sistemas produtores de água bruta abastecem a RMSP: Cantareira, Guarapiranga, Alto Tietê, Rio Grande, Rio Claro e Cotia (CASTRO, 2003; ANA, 2013). O Sistema Cantareira, que é responsável por cerca de metade do abastecimento da RMSP, deriva água da bacia do Rios Piracicaba, Capivari e Jundiaí (PCJ) para a bacia do Rio Tietê por uma série de reservatórios, canais e túneis. O sistema é composto por 4 reservatórios principais, sendo que três deles, Jaguari/Jacareí, Cachoeira e Atibainha estão localizados na bacia do PCJ (Figura 4) e o reservatório Paiva Castro pertence à bacia do Rio Tietê. Os dados operacionais, valores de demandas (tanto para a bacia do Rio Piracicaba quanto para a RMSP), vazóes mínimas e máximas (vazóes de restrição) a jusante dos reservatórios, foram compilados de Castro (2003), DAEE (2009) e ANA (2013). O resumo compilado é apresentado na Tabela 1 . As séries de vazóes afluentes aos reservatórios utilizada neste artigo foram obtidas de ANA (2013; 2014).

Os primeiros reservatórios do sistema, conforme Figura 4, são o Jaguari Jacareí que, conectados por um canal e que por este motivo podem ser analisados como um único reservatório. Estes são conectados ao reservatório Cachoeira pelo Túnel 7. O Túnel 6 conecta o reservatório Cachoeira ao Atibainha, que por sua vez é conectado ao Paiva Castro pelo Túnel 5 , onde ocorre a transferência entre as bacias do PCJ e Alto Tietê. Os três túneis citados transferem a água por gravidade. Do reservatório Paiva Castro, a água é conduzida por gravidade até a estação elevatória Santa Inês (EESI). Após ser elevada por um conjunto de bombas, a água passa por um pequeno reservatório para fins de regulação em caso de emergências (ex: falta de energia elétrica) e, finalmente conduzida à estação de tratamento de água bruta (ETA) Guaraú.

\section{Ajuste do modelo Chuva-Vazão}

Para esta análise foram consideradas as bacias hidrográficas, que contribuem aos três reservatórios principais localizados na bacia do PCJ: Jaguari/Jacareí,

TABELA 1

Resumo dos dados Operacionais e resumo das vazões mínimas e máximas a jusante do Sistema Cantareira

\begin{tabular}{|c|c|c|c|c|c|c|c|c|c|}
\hline \multirow[t]{2}{*}{ Reservatório } & \multicolumn{2}{|c|}{$\begin{array}{c}\text { Níveis } \\
\text { operacionais }(\mathrm{m})\end{array}$} & \multicolumn{3}{|c|}{ Volumes $\left(\mathrm{hm}^{3}\right)$} & \multirow{2}{*}{$\begin{array}{l}\text { Q Mín. } \\
\text { Jusante } \\
\left(\mathrm{m}^{3} / \mathrm{s}\right)\end{array}$} & \multirow{2}{*}{$\begin{array}{l}\text { * Q Máx. } \\
\text { Jusante } \\
\left(\mathrm{m}^{3} / \mathrm{s}\right)\end{array}$} & \multirow{2}{*}{$\begin{array}{c}\text { Área da } \\
\text { Bacia }\left(\mathrm{km}^{2}\right)\end{array}$} & \multirow{2}{*}{$\begin{array}{c}\text { Q Méd. } \\
\text { Hist. }\left(\mathrm{m}^{3} / \mathrm{s}\right)\end{array}$} \\
\hline & Mínimo & Máximo & Mínimo & Máximo & Útil & & & & \\
\hline Jaguari-Jacareí & 820,80 & 844,00 & 239,45 & 1047,49 & 808,04 & 1,00 & 50,00 & 1230,00 & 25,20 \\
\hline Cachoeira & 811,72 & 821,78 & 46,92 & 115,71 & 68,79 & 1,00 & 7,00 & 392,00 & 8,50 \\
\hline Atibainha & 781,88 & 786,86 & 199,20 & 295,52 & 96,32 & 1,00 & 3,00 & 312,00 & 6,00 \\
\hline \multirow[t]{2}{*}{ Paiva Castro } & 743,80 & 745,61 & 25,52 & 33,05 & 7,53 & 1,00 & 50,00 & 639,00 & 4,60 \\
\hline & & & & & & & & Total & 44,30 \\
\hline
\end{tabular}

* Vazão máxima a jusante: o valor máximo determinado pela portaria do DAEE (2009), vazões de restrição. 


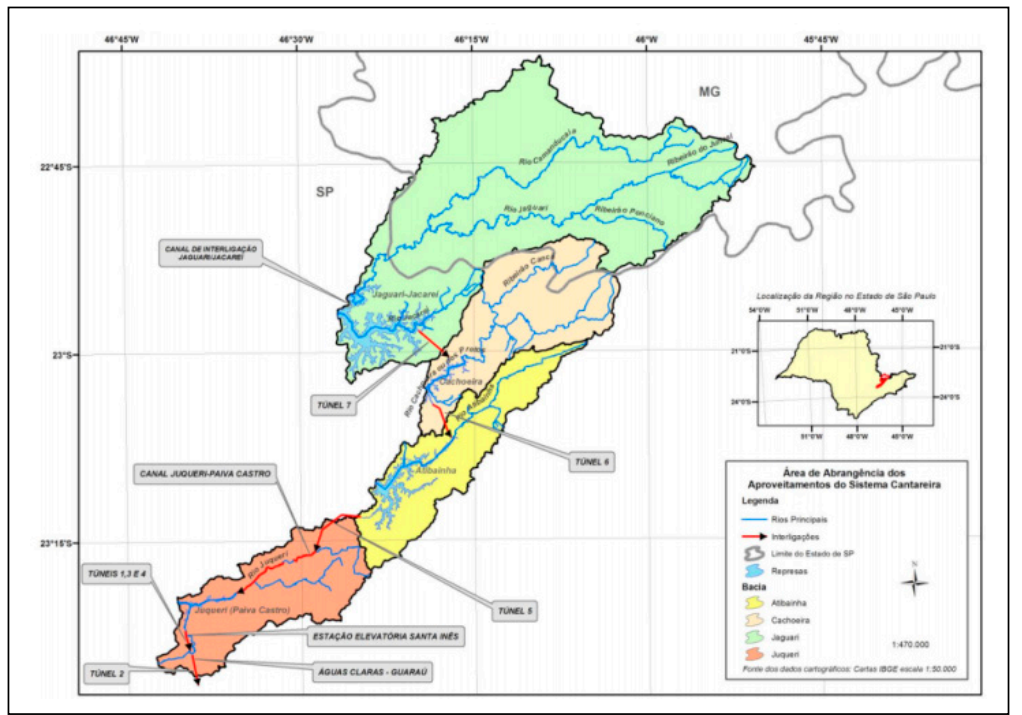

FIGURA 4. Localização e

Componentes Principais do Sistema Cantareira. Fonte: Sabesp (2009)

Cachoeira e Atibainha. A calibração e verificação do modelo chuva-vazão SMAP foi realizado no período de dados históricos de 1993 a 2004 utilizando dados de precipitação histórica mensal disponibilizados no Hidroweb da Agência Nacional de Águas - ANA. A Tabela 2 apresenta a lista de postos utilizados e localizados na regiáo do estudo. $\mathrm{O}$ critério para seleção dos postos foi a disponibilidade de dados consistentes no período de 1952 a 2004 , período utilizado para correçáo dos modelos climáticos.

A partir destes postos, as séries de precipitação mensal em cada uma das bacias foram calculadas através do método dos polígonos de Thiessen, no qual é determinada a área de influência de cada posto em cada uma das bacias para cada reservatório analisado.
Embora eventos de chuva dificilmente sejam uniformes em toda bacia de drenagem, por se tratar de análise mensal, é possível assumir que a metodologia aplicada possui representatividade satisfatória da precipitação.

As séries de precipitação para os cenários futuros foram calculadas utilizando 16 modelos climáticos listados na Tabela 3. A lista apresenta os dados básicos de cada um dos modelos: país de origem, código, centro desenvolvedor, o número de rodadas disponíveis e a resolução horizontal em graus decimais.

Dos modelos selecionados, os três cenários futuros analisados foram: RCP 2.6, RCP 4.5 e RCP 8.5 sendo que, para cada cenário, uma ou mais rodadas estão disponíveis e utilizadas. Os dados destes e outros

TABELA 2

Lista de postos pluviométricos utilizados para cálculo da precipitação histórica observada em cada sub-bacia

\begin{tabular}{|c|l|c|c|c|}
\hline Código & Nome do Posto & Altitude & Início & $\begin{array}{c}\text { Dados } \\
\text { (anos) }\end{array}$ \\
\hline 2346094 & Piracaia & 790 & $03 / 1942$ & 67 \\
\hline 2245050 & Sao Francisco Xavier & 730 & $05 / 1939$ & 70 \\
\hline 2246035 & Vargem (US. Flores - CPFL) & 940 & $08 / 1937$ & 71 \\
\hline 2246090 & Joanopolis & 920 & $06 / 1951$ & 57 \\
\hline 2346010 & Nazare Paulista & 790 & $06 / 1946$ & 62 \\
\hline 2346021 & Franco Da Rocha & 740 & $04 / 1937$ & 72 \\
\hline 2346030 & Bom Sucesso & 770 & $08 / 1936$ & 72 \\
\hline
\end{tabular}


TABELA 3

Lista de modelos climáticos analisados

\begin{tabular}{|l|l|l|c|c|}
\hline \multirow{2}{*}{ País } & Modelo & Nome do Centro & Número de & Resolução \\
\cline { 4 - 5 } & & & $\begin{array}{c}\text { Rodadas. vs } \\
\text { Lat.) }\end{array}$ \\
\hline China & bcc_csm_1_1 & Beijing Climate Center, China Meteorological Administration & 1 & $2.8 \times 2.8$ \\
\hline China & BNU-ESM & Collegeof Global Changeand Earth System Science & 1 & $2.8 \times 2.8$ \\
\hline Canada & CanESM2 & Canadian Centre for Climate Modeling and Analysis & 5 & $2.8 \times 2.8$ \\
\hline USA & CCSM4 & National Center of Atmospheric Research & 1 & $1.25 \times 0.94$ \\
\hline France & CNRM-CM5 & National Centre of Meteorological Research & 1 & $1.4 \times 1.4$ \\
\hline Australia & CSIRO-Mk3-6-0 & $\begin{array}{l}\text { Commonwealth Scientificand Industrial Research Organization/ } \\
\text { Queensland Climate Change Centre of Excellence }\end{array}$ & 10 & $1.8 \times 1.8$ \\
\hline China & FGOALS-g2 & $\begin{array}{l}\text { LASG, Institute of Atmospheric Physics, Chinese Academy of } \\
\text { Sciences }\end{array}$ & 1 & $2.8 \times 2.8$ \\
\hline USA & GFDL-CM3 & NOAA Geophysical Fluid Dynamics Laboratory & 1 & $2.5 \times 2.0$ \\
\hline USA & GFDL-ESM2G & NOAA Geophysical Fluid Dynamics Laboratory & 1 & $2.5 \times 2.0$ \\
\hline France & IPSL-CM5A-LR & Institute Pierre Simon Laplace & 4 & $3.75 \times 1.8$ \\
\hline Japan & MIROC5 & $\begin{array}{l}\text { Atmosphere and Ocean Research Institute (The University of } \\
\text { Tokyo), National Institute for Environmental Studies, and Japan } \\
\text { Agency for Marine-Earth Science and Technology }\end{array}$ & 3 & $1.4 \times 1.41$ \\
\hline Japan & MIROC-ESM & $\begin{array}{l}\text { Japan Agency for Marine-Earth Science and Technology, } \\
\text { Atmosphere and Ocean Research Institute (The University of } \\
\text { Tokyo), and National Institute for Environmental Studies }\end{array}$ & 1 & $2.8 \times 2.8$ \\
\hline Germany & MPI-ESM-LR & Max Planck Institute for Meteorology & 3 & $1.88 \times 1.87$ \\
\hline Japan & MRI-CGCM3 & Meteorological Research Institute & 1 & $1.1 \times 1.1$ \\
\hline Norway & NorESM1-M & Norwegian Climate Center & $2.5 \times 1.9$ \\
\hline United & HadGEM2-ES & Met Office Hadley Centre & $1.25 \times 1.875$ \\
\hline
\end{tabular}

modelos são disponibilizados em (http://cmip-pcmdi. llnl.gov/cmip5) no formato NetCDF (UNIDATA, 2014). Os dados foram extraídos dos arquivos NetCDF com auxílio de uma biblioteca específica para tal (escrita em $\mathrm{C}++$ ), combinada em código desenvolvido em C\# (C Sharp).

Para a análise do possível impacto na garantia de fornecimento do Sistema Cantareira, foram analisadas as curvas de frequência das séries determinadas pela metodologia apresentada. Todos os resultados dos cenários futuros (RCP 2.6, RCP 4.5 e RCP 8.5) foram combinados na análise uma vez que é incerto o cenário que deverá ocorrer. Desta forma, os resultados apresentados compreendem mais de 120 séries de precipitaçóes e por consequência possibilitam gerar o mesmo número de séries de vazóes com a aplicação do modelo SMAP. É importante ressaltar também que a análise foi dividida em período úmido e período seco. O período úmido considerado foram os meses de Outubro à Março e o período seco de Abril à Setembro.

\section{RESULTADOS E DISCUSSÃO}

Na Figura 5 e Figura 6sãoapresentadas as curvas de permanência das precipitaçóes corrigidas dos modelos climáticos para os períodos úmido e seco, respectivamente para a bacia do reservatório Jaguari/ Jacareí. As curvas de permanência apresentadas em tons de azul representam os modelos climáticos e a 
curva de permanência baseada na série de precipitação histórica observada é destacada em cor vermelha. Ambos os gráficos combinam os resultados de cerca de 120 séries dos modelos climáticos e é possível observar a grande incerteza a eles associada. Nota-se ainda que existe uma leve tendência de aumento das precipitaçóes em relação à média histórica, bem como amplificação dos extremos, tanto precipitaçóes máximas quanto mínimas.

Nas figuras Figura 7 a Figura 9 são apresentadas os boxplot das vazóes médias calculadas pelos modelos climáticos, as vazões mínimas e máximas dos GCMs comparando com o média das vazóes afluentes históricas observadas para os reservatórios Jaguari/Jacareí, Cachoeira e Atibainha, respectivamente.

Para o reservatório Jaguari/Jacareí existe uma tendência de aumento da vazáo para a maioria dos modelos, conforme gráfico boxplot das vazóes médias para cada mês, conforme Figura 7, comparada à vazáo da média histórica observada. Nota-se que para os meses úmidos (Outubro a Fevereiro) existe tendência de aumento significativo na vazão, em média de 10 a $20 \%$ em relação à média histórica, enquanto nos meses secos essa anomalia não é tão significativa.

Nos outros dois reservatórios, Cachoeira e Atibainha, observa-se queda na vazão afluente calculada utilizando a metodologia proposta comparando à média histórica observada, conforme apresentado nos gráficos das Figuras 8 e 9, respectivamente. De

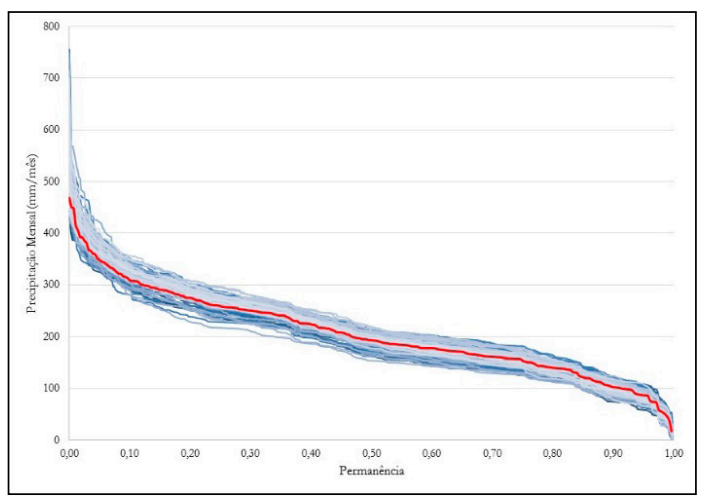

FIGURA 5. Curvas de permanência da precipitação para os meses úmidos obtidos dos modelos climáticos em tons de azul comparadas à curva de permanência da série histórica observada (linha em vermelho) para a Bacia do Reservatório Jaguari/ Jacareí forma simular ao observado na bacia do reservatório Jaguari/Jacareí

No gráfico da Figura 10 é apresentado um boxplot das vazões mínimas dos modelos climáticos para cada um dos meses do ano, comparando com as vazôes afluentes do ano 2013/2014 para a bacia hidrográfica do reservatório Jaguari/Jacareí. Esta comparação foi realizada pelo fato da atual crise hídrica, que passa o Sistema Cantareira, sendo a pior de sua história e as vazóes afluentes médias mensais são as mais baixas já registradas. Os resultados apresentados no gráfico, mostram que existe possibilidade, com base nos resultados dos GCMs, deste cenário se repetir no horizonte analisado. A crise atual está sendo causada pelos baixos níveis de precipitação, especialmente nos meses úmidos, no qual os níveis do sistema usualmente se recuperam.

\section{CONCLUSÃO}

Neste artigo, é apresentada uma análise do possível impacto de mudanças climáticas na disponibilidade hídrica sobre um sistema de recursos hídricos voltado ao abastecimento público. A metodologia consiste em corrigir as séries de precipitaçáo mensal e evaporação média mensal disponibilizadas pelos modelos de circulação global (GCM) para cenários futuros, tomando como base dados históricos observados. A correção é realizada utilizando-se um método

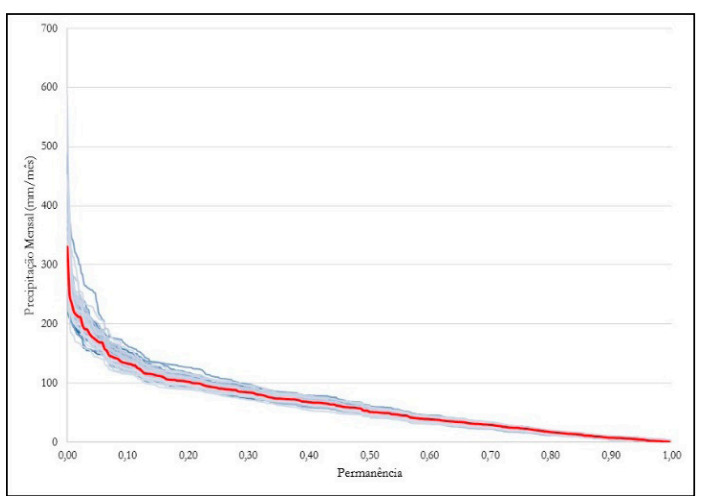

FIGURA 6. Curvas de permanência da precipitação para os meses secos obtidos dos modelos climáticos em tons de azul comparadas à curva de permanência da série histórica observada (linha em vermelho) para a Bacia do Reservatório Jaguari/ Jacareí 


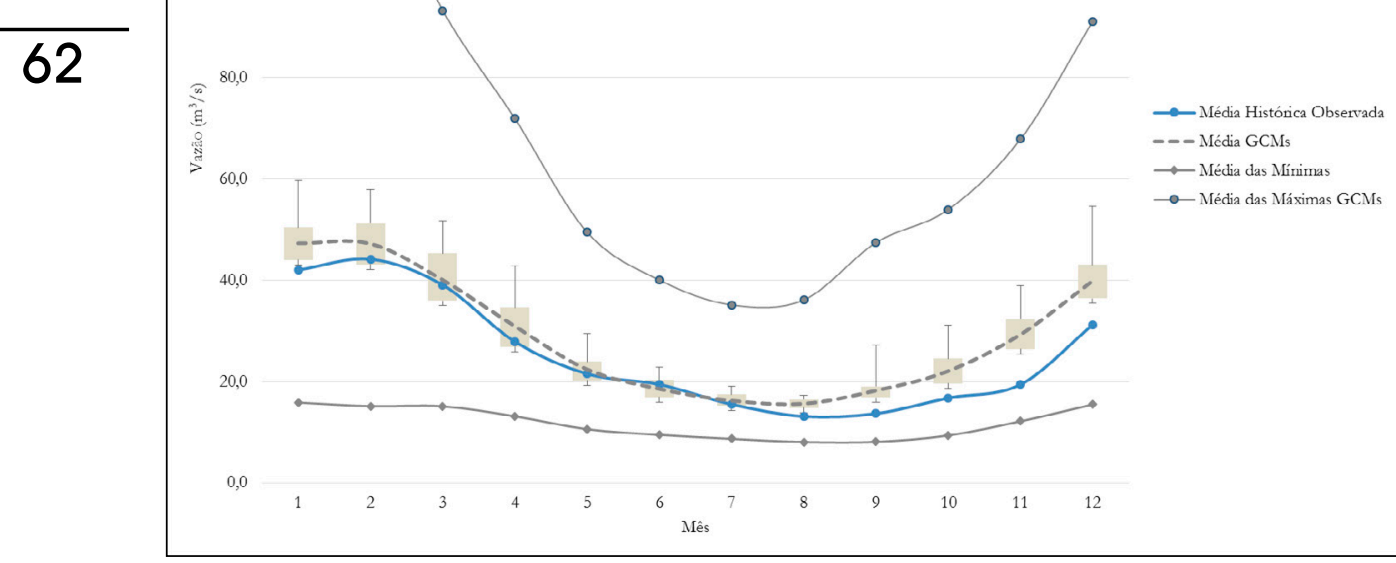

FIGURA 7. Boxplot da vazão calculada combinando todos os cenários futuros à média histórica (linha em azul) para a Bacia do Reservatório Jaguari/Jacareí

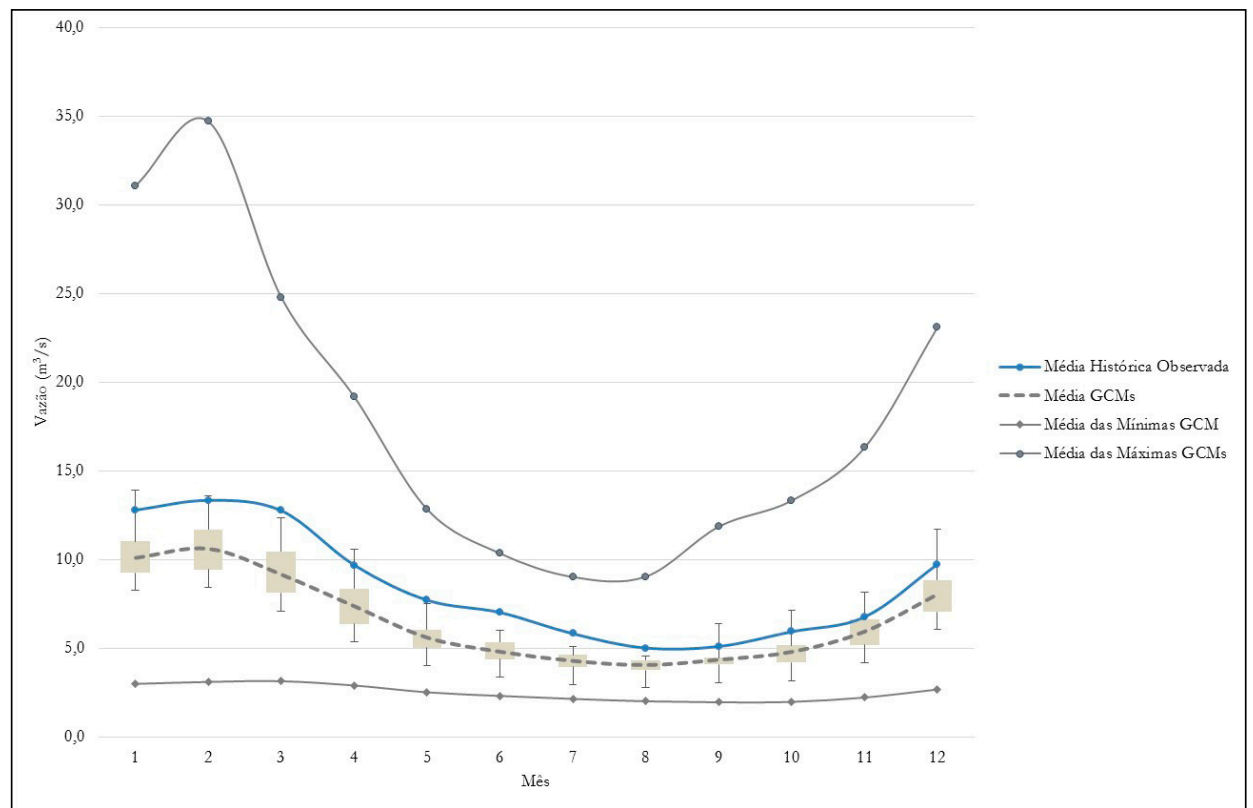

FIGURA 8. Boxplot da vazão calculada combinando todos os cenários futuros à média histórica (linha em azul) para a Bacia do Reservatório Cachoeira 


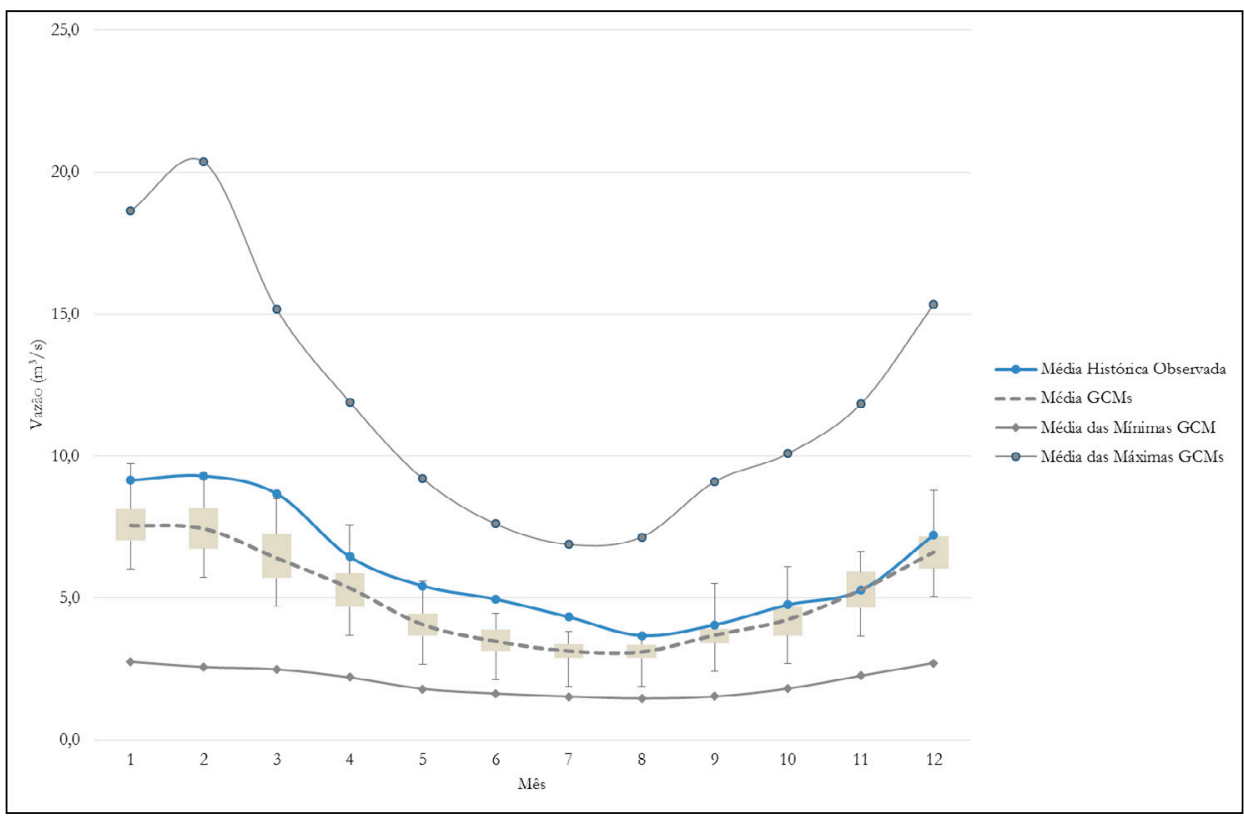

FIGURA 9. Boxplot da vazão calculada combinando todos os cenários futuros à média histórica (linha em azul) para a Bacia do Reservatório Atibainha

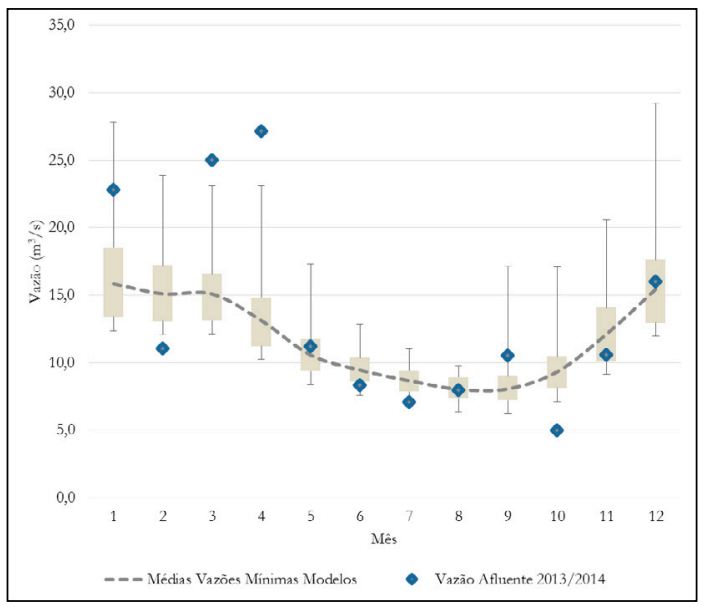

estatístico, com o qual procurar-se corrigir o erro sistemático embutido nos modelos climáticos.

A metodologia sugerida foi aplicada às bacias de contribuição dos principais reservatórios do Sistema Cantareira, responsável por abastecer cerca de $50 \%$ da RMSP. Dados de postos pluviométricos da regiáo de estudo foram utilizados para representar a
FIGURA 10. Boxplot das vazões mínimas dos modelos climáticos e a vazão afluente da bacia do reservatório Jaguari/Jacareí do período seco de 2013/2014.

precipitação média sobre as bacias de drenagem dos principais reservatórios do sistema. Foram analisados três cenários futuros de 16 (dezesseis) modelos climáticos, RCP 2.6, 4.5 e 8.5 e todas as rodadas disponíveis em cada um dos modelos, totalizando mais de 120 séries de precipitação mensal. Os dados de precipitaçáo extraídos dos modelos de clima 
foram corrigidos com uma metodologia descrita e um modelo chuva-vazão utilizado para obter as séries de vazóes para o período futuro. Com a utilização de 16 modelos climáticos, os resultados apresentam grandes incertezas. Entretanto, nota-se que, analisando apenas as séries de precipitaçóes, existe a possibilidade de aumento dos eventos extremos, especialmente nos meses úmidos, maior número de meses com níveis mais baixos que a média histórica observada e mais eventos ou meses com precipitação acima da média observada.

A metodologia apresentada para o cálculo das afluências e correção das séries de precipitação é de fácil aplicação e possui baixo custo em termos de recursos computacionais. Neste artigo, foram utilizados modelos de circulaçáo global, entretanto a mesma metodologia pode ser aplicada a modelos com precisão espacial e temporal mais detalhada, como é o caso de modelos climáticos regionais.

\section{AGRADECIMENTOS}

Os autores agradecem o apoio financeiro do $\mathrm{CNPq}$ (Conselho Nacional de Pesquisa e Desenvolvimento) ao primeiro autor concedido sob forma de bolsa de estudos.

\section{Referências}

ANA Agência Nacional de Águas. Dados de Referência Acerca da Outorga do Sistema Cantareira, Brasília (DF), (2013).

ANA Agência Nacional de Águas. Renovação da Outorga do Sistema Cantareira, 2014. Disponivel em http://www2.ana.gov.br/Paginas/ servicos/outorgaefiscalizacao/renovacaocantareira.aspx. acesso em 30/Setembro/2014.

ALLAN, R. P.; SODEN, B. J. Atmospheric warming and the amplification of precipitation extremes. Science, v.321, p.1480-1484, 2008.

BOWERING, E. A.; PECK, A. M.; SIMONOVIC, S .P. A flood risk assessment to municipal infrastructure due to changing climate part I: Methodology, Urban Water Journal, DOI:10.1080/1573062X.2012.758293, 2013.

CASTRO, H. L. Avaliação da disponibilidade hídrica para abastecimento público da Região Metropolitana de São Paulo com o uso de um SSD - Sistema de Suporte a Decisões. 2013, 169p. Dissertação (Mestrado). Universidade de São Paulo, São Paulo.

BRAVO, J. M.; COLLISCHONN, W.; DA PAZ, A.R.; ALLASIA, D.; DOMECQ, F. Impact of projected climate change on hydrologic regime of the Upper Paraguay River basin. Climatic Change, DOI10.1007/s10584-013-0816-49 2, 2013.

Cunha, V. Estudo para Proposta de Critérios de Qualidade da Água para Reúso Urbano. Tese de Doutorado. 2008, 94 p. Universidade de São Paulo, São Paulo, SP.

Cunderlik, J. M. e Simonovic, S. P. Hydrological extremes in a southwestern Ontario river basin under future climate conditions. Hydrological Sciences Journal. v.50, n.4, p.631-654, 2005.

DAEE Secretaria De Estado De Saneamento E Energia Departamento De Águas E Energia Elétrica Diretoria De Procedimentos De Outorga E Fiscalização. Nota Técnica DAEE/DPO - Sistema Cantareira - Recomendações para manutenção de volumes de espera nos reservatórios dos aproveitamentos do Sistema Cantareira. 2009.

DAS, S.; SIMONOVIC, S.P. Assessment of uncertainty in flood flows under climate change - the Upper Thames River basin (Ontario, Canada), British Journal of Environment and Climate Change, v.2, n.4, p.318-338, 2012.

EUM, H.-I.; SIMONOVIC, S. P. Assessment on variability of extreme climate events for the Upper Thames River basin in Canada. Hydrological Processes, v.26, n.4, p.485-499. doi:10.1002/hyp.8145, 2012.

IPCC Climate Change 2007: The Physical Science Basis. Contribution of Working Group I to the Fourth Assessment Report of the Intergovernmental Panel on Climate Change [Solomon, S., D. Qin, M. Manning, Z. Chen, M. Marquis, K.B. Averyt, M. Tignor e H.L. Miller (eds.)]. Cambridge University Press, Cambridge, United Kingdomand New York, NY, USA, 2007. 996 p.

IPCC Summary for Policymakers. In: Climate Change: The Physical Science Basis. Contribution of Working Group I to the Fifth Assessment Report of the Intergovernmental Panel on Climate Change [Stocker, T.F., D. Qin, G.-K. Plattner, M. Tignor, S.K. Allen, J. Boschung, A. Nauels, Y. Xia, V. Bexand P.M. Midgley (eds.)]. Cambridge University Press, Cambridge, United Kingdomand New York, NY, USA, 2014.

GAUR, A.; SIMONOVIC, S. P. Climate Change Impact on Flood Hazard in the Grand River Basin, Ontario, Canada. Water Resources Research Report no. 084, Facility for Intelligent Decision Support, Department of Civil and Environmental Engineering, London, Ontario, Canada, 2013, 92 p. ISBN: (print) 978-0-7714-3063-3; (online) 978-0-7714-3064-0.

KHARIN, V.V.; ZWIERS, F.W.; ZHANG, X.; HEGERL, G. Changes in temperature and precipitation extremes in the IPCC ensemble of global coupled model simulations. Journal of Climate, v.20, p.1519-1444, 2013.

LOPES, J. E. G.; BRAGA, B. P. F.; CONEJO, J. G. L. SMAP: A simplified hydrologic model. In: Applied Modeling in Catchment Hydrology Water Resources Publications, Littleton, Co. 1982, p. 167-176.

Li, H.; Sheffield, J.; Wood, E. F. Bias correction of monthly precipitation and temperature fields from Intergovernmental Panel on Climate Change AR4 models using equidistant quantile matching. Journal of Geophysical Research, v.115(D10), D10101. 2010, doi:10.1029/2009JD012882. 
MARENGO, J. A., CHOU, S. C., KAY, G., ALVES, L. M., PESQUERO, J. F., SOARES, W. R., TAVARES, P. Development of regional future climate change scenarios in South America using the Eta CPTEC/HadCM3 climate change projections: climatology and regional analyses for the Amazon, São Francisco and the Paraná River basins. Climate Dynamics, v.38, n.9-10, p.1829-1848, 2011. doi:10.1007/s00382-011-1155-5 NAKICENOVIC N.; SWART R. (Eds.) IPCC Special Report on Emission Scenarios. Cambridge University Press, UK. 2000, 570 p.

PIANI, C.; WEEDON, G. P.; BEST, M.; GOMES, S. M.; VITERBO, P.; HAGEMANN, S.; HAERTER, J. O. Statistical bias correction of global simulated daily precipitation and temperature for the application of hydrological models. Journal of Hydrology, v.395, n.3-4, p.199-215, 2010). doi:10.1016/j.jhydrol.2010.10.024.

PRICE, K. V.; STRONG, R. M.; LAMPINEN, J. A. Differential evolution: A practical approach to global optimization. Berlin: Springer. 2005. Price, K. V.; STORN, R. M. Differential evolution - A simple evolution strategy for fast optimization. Dr. Dobb's Journal. v.22, p.18-24. 1997. SABESP Companhia de Saneamento Básico do Estado de São Paulo. Estudos de Cheias para Avaliação dos Volumes de Espera dos Aproveitamentos do Sistema Cantareira, São Paulo - SP. 2009.

SALATHÉ, JR. E. P.; MOTE, P. W.; WILEY, M. W. Considerations for selecting downscaling methods for integrated assessments of climate change impacts. International Journal of Climatology, v.27, p.1611-1621, 2007.

SCHARDONG, A. et al. comparação de algoritmos evolucionários na calibração de modelo chuva-vazão - SMAP. In: XVIII Simpósio Brasileiro de Recursos Hídricos, 2009, Campo Grande - MS. XVIII Simpósio Brasileiro de Recursos Hídricos. Porto Alegre : ABRH.

SCHARDONG, A.; SIMONOVIC, S. P.; VASAN, A. A multi-objective evolutionary approach to optimal reservoir operation, ASCE Journal of Computing in Civil Engineering, v.27, n.2, p.139-147, 2013.

SHARIF, M.; BURN, D. H.; WEY, K. M. Daily and hourly weather data generation using a k-nearest neighbour approach, 18th Canadian Hydrotechnical Conference. p.1-10, 2007.

SOLAIMAN, T. A.; KING, L. M.; SIMONOVIC, S. P. Extreme precipitation vulnerability in the Upper Thames River basin: uncertainty in climate model projections. International Journal Climatology, v.31, p.2350-2364, 2011, doi:10.1002/joc.2244

TAYLOR, K.E.; STOUFFER, R. J.; MEEHL, G.A. An overview of CMIP5 and the experiment design. Bulletin of the American Meteorological Society, v. 93, n.4, p.485-498, 2012. doi:10.1175/bams-d-11-00094.1.

UNIDATA, netCDF Documentation, 2014. Disponivel em http://www.unidata.ucar.edu/software/netcdf/docs/ acesso em 30/Setembro/2014. USACE. Hydrologic Modeling System HEC-HMS. User's Manual, Version 2.1. US Army Corps of Engineers, Hydrologic Engineering Center, 2001, 178 p.

VUUREN, D. P.; EDMONDS, J.; KAINUMA, M.; RIAHI, K.; THOMSON, A.; HIBBARD, K.; ROSE, S. K. The representative concentration pathways: an overview. Climatic Change, v.109, n.1-2, p.5-31, 2011. doi:10.1007/s10584-011-0148-z

WILCOX, E.M.; DONNER, L.J. The frequency of extreme rain events in satellitera in-rate estimates and an atmospheric general circulation model. Journal of Climate, v.20, n.1, p.53-69, 2007.

WCRP Coupled Model Intercomparison Project - Phase 5 Special Issue of the CLIVAR Exchanges Newsletter, No. 56, v. 15, n. 2, 2011.

Andre Schardong Postdoctoral Fellow, Department of Civil and Environmental Engineering, University of Western Ontario, London, Ontario, Canada, N6A 5B9. E-mail: aschardo@uwo.ca.

Slobodan P. Simonovic Professor, Department of Civil and Environmental Engineering, University of Western Ontario, London, Ontario, Canada, N6A 5B9. E-mail: simonovic@uwo.ca.

Joaquin I. B. Garcia Professor, Departamento de Engenharia Hidráulica e Ambiental, Universidade de São Paulo, SP, CEP: 05068-010. E-mail: joaquinbonne@gmail.com. 
